\title{
Estimation of Evocation of Friendship Based on Similarity of Pulse Rate Variability of Users for Event-based Social Networks
}

\author{
Yusuke Kajiwara, ${ }^{*}$ Yuki Kubo, ${ }^{2}$ and Haruhiko Kimura ${ }^{1}$ \\ ${ }^{1}$ Department of Production Systems Engineering and Sciences, Komatsu University, \\ 1-3 Shichomachi nu, Komatsu, Ishikawa 923-8511, Japan, \\ ${ }^{2}$ College of Information Science and Engineering, Ritsumeikan University, \\ 1-1-1 Noji-higashi, Kusatsu, Shiga 525-8577, Japan
}

(Received October 17, 2017; accepted December 11, 2017)

Keywords: friendship, similarity of pulse rate variability of users, favorability, machine learning

In contrast to traditional social network services (SNSs), event-based social networks determine close friendships (CFs) of users who share experiences and emotions with candidate friends in offline events. However, we could not provide feedback to cyberspace regarding the place, time, and target of a user realizing friendship since there is no technique for conveniently measuring the evocation of friendship during offline events. In this research, we propose a method of estimating the evocation of friendship using the similarity in the pulse rate variabilities (PRVs) of users when empathy is evoked between them. The user can be made aware of friendship estimated automatically through machine learning by wearing a wristwatch-type pulsimeter. CFs are more likely to evoke empathy than superficial friendships (SFs). To demonstrate the usefulness of this method, we conducted an experiment assuming an event where a group of four people are enjoying their time in an amusement park. From the experimental results, we showed that the similarity of the PRVs in CFs is greater than that in SFs when the favorability rating is high and the users like each other. Moreover, we showed that the proposed method estimated the evocation of friendship during the attraction experience with an $f$-measure of 0.74 at maximum and during an offline event with a mean $f$-measure of 0.78 . The results showed the usefulness and effectiveness of this method.

\section{Introduction}

Most people use social network services (SNSs) for information exchange and friendship. ${ }^{(1)}$ Friendship is defined as a desire to maintain a sustained connection with others. ${ }^{(2)}$ Candidate friends are recommended on the basis of personal characteristics such as the user's participation rate, ${ }^{(3)}$ common interests, ${ }^{(4)}$ positional relationship, ${ }^{(5)}$ and lifestyle. ${ }^{(6)}$ However, it is difficult for companions in cyberspace to satisfy some of the elements (sharing experiences and emotions; developing trusting relationships) ${ }^{(7,8)}$ needed to become friends from candidate friends. Therefore, users are not always able to become friends with recommended candidate friends.

As SNSs can deal with this problem, an application of event-based social networks (EBSNs), *Corresponding author: e-mail: kajiwararesearchbox68@gmail.com http://dx.doi.org/10.18494/SAM.2018.1777 
such as Eventbrite, Meetup, and Plancast, has been developed recently to promote community formation by correlating cyberspace and the real world. EBSNs promote casual gatherings for watching movies, going to amusement parks, and parties, and other events. ${ }^{(9)}$ In contrast to traditional SNSs, EBSNs cultivate close friendships (CFs) among users through the sharing of experiences and emotions with candidate friends at offline events. However, we could not provide feedback to cyberspace regarding the place, time, and target of the user realizing friendship since there is no technique for conveniently measuring the evocation of friendship during offline events. Therefore, we could not correlate friendship in cyberspace with that in the real world.

In related works, friendship is often measured using parameters such as favorability rating, ${ }^{(10)}$ McGill Friendship Questionnaire-Respondent's Affection (MFQ-RA), and McGill Friendship Questionnaire-Friendship Function (MFQ-FF). ${ }^{(1)}$ However, when there are many EBSN participants, the use of a questionnaire places a heavy burden on the user.

In this research, we propose a method of estimating friendship on the basis of the similarity in the pulse rate variabilities (PRVs) of users when empathy is evoked between them. Empathy means that the user can predict the feelings of others and can feel the same emotions. ${ }^{(12)}$ When a friendship is superficial, empathy is evoked only for negative emotion. When a friendship is close, empathy is evoked for both negative and positive emotions. ${ }^{(13)}$ Therefore, friendship can be estimated by how many times empathy is evoked.

Empathy has been measured in laboratory experiments using brain activity ${ }^{(14-16)}$ and the heartbeat evoked potential (HEP) amplitude measured with an electrocardiogram. ${ }^{(17)}$ However, such measurements are difficult to use during an event since electroencephalographs and electrocardiograms are expensive and the equipment is large, which place economical and physical burdens on the users. Therefore, in this research, we use a wristwatch-type pulsimeter that is inexpensive, compact, and already popularly used. Users can evaluate the friendship automatically using the wristwatch-type pulsimeter on their arm. When empathy is evoked between users, users feel the same emotion. Emotion can be measured as heart rate variability (HRV). Emotions such as delight and surprise activate the sympathetic nerve and increase the heart rate (HR). ${ }^{(18)}$ Emotions that promote relaxation activate the parasympathetic nerve and decrease the HR. In addition, when user actions are synchronized, the favorability rating between users is increased. ${ }^{(19)}$ User actions increase the HRV of the user. Therefore, the HRV is similar to that when empathy is evoked between users and the user actions are synchronized. PRV approximates HRV. The similarity in PRVs is described by the correlation coefficient of the pulse rates (PRs) of users. We estimate friendship by inputting the similarity in the PRV of users into a machine learning algorithm.

To demonstrate the usefulness of this method, we conducted an experiment assuming an event where a group of four people are enjoying their time in an amusement park. As the four people experienced an attraction in the amusement park, we measured their emotion, favorability toward each user, and PRs. The emotion and favorability toward each user were obtained using a questionnaire. PR has a positive correlation with exercise, making it necessary to consider the increase in PR owing to walking, since the user walks to the next attraction after experiencing an attraction in the amusement park. To verify the influence of walking, the 
subject was for the first time put a smartphone in the pants pocket and a pedometer application was used to obtain the acceleration and travel time of walking. To show that friendship can be estimated from the similarity in PRs, one friend and two other people to be met for the first time were placed in the group. The two friends had a closer friendship than the other firsttimers. In this research, we defined friends as people with relationships built on CFs and define the relationship of people who had met for the first time as a relationship built on superficial friendships (SFs). We verified a significant difference in the similarity in the PRVs between CFs and SFs. We confirmed that the increase in PR owing to walking had little influence on the PRVs during the attraction experience. We showed that the similarity in the PRVs of users during the attraction experience was high when users had favorability toward each other. There was a weak positive correlation between the favorability rating and the similarity in the PRVs during the attraction experience. The similarity in the PRVs of CFs was higher than that of SFs when the favorability rating was high and the users had favorability toward each other. The experimental results indicated that it is difficult to estimate the friendship between user $i$ and user $j$ from the questionnaire results on favorability and emotions. The evocation of friendship during the attraction experience was estimated to have an $f$-measure of 0.74 at maximum from the similarity in the PRV of users at each attraction and the evocation of friendship during the offline event was estimated to be a mean $f$-measure of 0.78 from the mean similarity in the PRVs of users at the offline event. These results showed the usefulness and effectiveness of the method.

In Sect. 2, we describe a related work on the process of friendship, a method of measuring empathy, and a method of measuring friendship. In Sect. 3, we describe an algorithm for estimating friendship from the similarity in the PRV of users. In Sect. 4, we describe the experimental environment and experimental method. In Sect. 5, we analyze the experimental results and state our findings on the relationship between the obtained similarity in PRVs, momentum, favorability, and empathy. In Sect. 6, we show the estimation accuracy of the evocation of friendship in each attraction at an amusement park or offline event. In Sect. 7, we show the contribution and limitations of this method referring to the results in Sects. 5 and 6. In Sect. 8, we give the conclusions and describe future works.

\section{Related Works}

\subsection{Definition of friendship}

Adams et $a l .{ }^{(7)}$ described friendship in the process of action, the cognitive process, the emotional process, the unity, the uniformity, the frequency of contact, the length of acquaintance, and the contact continuity. Humans recognize themselves as friends on the basis of common interests and trust. Emotional sharing has been shown to be more important than behavior and cognition. It was shown that there is a gender difference in the process of friendship. Women consider the quality of feelings important in the process of friendship. Men consider the frequency of contact and the length of friendship important in the process of friendship. McFadden et al. ${ }^{(20)}$ showed that there is a significant difference in emotional 
sharing between long-time friends and new friends. Long-time friends and new friends feel more comfortable sharing positive emotion than negative emotion. Friends increase mutual social support by sharing emotions. Sias et al. ${ }^{(8)}$ analyzed the transition from acquaintance to friends. The transition from a colleague to a friend is mainly due to approach and collaboration, sharing a common foundation, and socialization outside an organization. The transition from friends to close friends is mainly related to lifelong events, work-related problems, and the passage of time. Motomura et al. ${ }^{(13)}$ showed that empathy is different between a person you have met for the first time and a friend. A newly met person easily evokes empathy for negative emotion including the danger-sensing function, but not empathy for positive emotion. Friends experience empathy for both positive and negative emotions, and often synchronize behaviors.

In summary, friendship has gender and age differences, indicating that sharing experience and emotion with friends is an important factor in the transition from acquaintance to friends and from friends to close friends. Users with SF only evoke empathy for negative emotion. Users with CF evoke empathy for both negative and positive emotions. CF is more likely to evoke empathy than SFs. Therefore, friendship can be estimated from the number of times empathy is evoked.

\subsection{Relationship between empathy and biological signals}

Jackson et al. ${ }^{(14)}$ showed that humans show a feeling of pain when shown a picture of a person in pain. In addition, their front herpes zoster region, anterior nasal cavity, cerebellum, and hypothalamus are activated. Yang et $a l^{(15)}$ reported a gender difference in painful emotions related to the resonance of the sensory motor cortex of the brain. Decety et al. ${ }^{(16)}$ reported that there are age-related changes in the dorsal and ventral prefrontal cortices in characterizing developmental changes in the brain activity in neuronal circuits supporting empathy. Fukushima et al. ${ }^{(17)}$ reported that the self-report measurement of empathy is related to the HEP amplitude. Rakshit et al. ${ }^{(21)}$ identified three emotions, namely, happiness, sadness, and neutral, with an accuracy of $83.8 \%$ on average using a support vector machine (SVM) that learns HRV features. Emotions are estimated from the activity of the autonomic nervous. Frequency responses of R-R intervals are calculated as HRV. The frequency of HRV is divided into the high-frequency (HF) band and the low-frequency (LF) band. The frequency band from 0.15 to $0.45 \mathrm{~Hz}$ is called the HF band and represents the activity of the parasympathetic nerve. The frequency band from 0.04 to $0.15 \mathrm{~Hz}$ is called the LF band and represents the activity of the parasympathetic and sympathetic nerves. Emotions are estimated from the power spectra of these HF and LF bands.

The measurement of empathy from an electrocardiogram or electroencephalogram cannot be used in events such as visits to an amusement park since it places an economic and physical burden on the user. Humans sympathize with the same emotions predicted others through empathy. A related work showed that emotion can be estimated by measuring the activity of the sympathetic and parasympathetic nerves. ${ }^{(18)}$ The activation of the sympathetic and parasympathetic nerves is reflected in the PRV so that emotion can be estimated from the PRV. Therefore, empathy is described as a similarity in PRVs among users. 


\subsection{Relationship between behavioral synchronization and friendship}

Related works showed that behavioral synchronization between users enhances friendship ${ }^{(19)}$ and improve socialization. ${ }^{(22)}$ Zhao $^{(23)}$ revealed that friendship is enhanced by conversation with attention, appreciation of each other, self-disclosure, and smiling. Brambilla ${ }^{(24)}$ revealed that user's actions are often synchronized when the user's partners are sincere. When the synchronization of action declines, there is a high possibility of acting by partners who are uncooperative. Hove ${ }^{(19)}$ reported that the synchronization of action is an important factor contributing to the favorability rating. It is difficult to improve the favorabolity rating merely by imitating action. Zarrugh ${ }^{(25)}$ reported that energy consumption positively correlates with the magnitude of motion and exercise time.

For the above reasons, users synchronize their actions to increase the favorability rating. Pulse is increased by momentum. Therefore, the PRVs of users can be made similar to each other through behavioral synchronization.

\subsection{Measurement of friendship}

Mendelson ${ }^{(11)}$ created a questionnaire for measuring friendship. MFQ-RA is used to measure respondents' emotions on the basis of satisfaction with the friendship. MFQ-FF issued to measure the degree to which friends develop their friendship (friendship, help, intimacy, trusted alliance, self-verification, stimulating emotional security). Eagle ${ }^{(26)}$ inferred the levels of friendship on the basis of observational data showing temporal and spatial patterns of physical proximity and speech patterns acquired from mobile phones, with $95 \%$ accuracy.

When the number of questions in the questionnaire increases, the user finds it difficult to answer the questionnaire and an accurate result cannot be obtained. Eagle ${ }^{(26)}$ showed, using online information, that friendship cannot be measured in offline events.

\section{Estimation of Friendship from Similarity in PRV of Users}

Figure 1 shows the flow of estimating friendship from the similarity in the PRV of users when they empathize with each other. The user wears a wristwatch-type pulsimeter to measure the PR. The activities of the parasympathetic and sympathetic nerves are measured from the power spectra in the HF and LF bands. The wristwatch-type pulsimeter measures the PR from the blood flow rate. The PR approximates the HR. The blood flow rate respectively increases and decreases as the sympathetic and parasympathetic nerves are activated. Therefore, it is

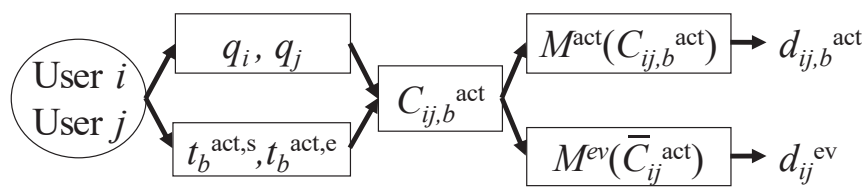

Fig. 1. Estimation of friendship using the similarity of user's PR. 
possible to indirectly measure the activities of the parasympathetic and sympathetic nerves by measuring the PR.

We define any user's PR at an arbitrary time as $q_{i}(t) . t$ is defined as an arbitrary time. $i$ is defined as a user. When the friendship between users is superficial, the users only feel empathy for negative emotions such as pain, anxiety, and fear. When the friendship between users is close, the users feel empathy for both negative and positive emotions. ${ }^{(13)}$ Therefore, the users are more likely to empathize with each other as they become closer. Emotion can be quantified by the PRV. Therefore, it is expected that $q_{i}(t)$ of user $i$ and $q_{j}(t)$ of user $j$ will be similar when empathy is evoked between them. When there is little empathy between users, the similarity in the PRVs of the users is small. When there is a high degree of empathy between users, the similarity in the PRVs of the users is large. The similarity between $q_{i}(t)$ and $q_{j}(t)$ is calculated using

$$
C_{i j, b}^{a c t}=\frac{\sum_{t=t_{b}^{a c t, s}}^{t_{b}^{a c t, e}}\left(q_{i}(t)-\overline{q_{i, b}^{a c t}}\right)\left(q_{j}(t)-\overline{q_{j, b}^{a c t}}\right)}{\sqrt{\sum_{t=t_{b}^{a c t, s}}^{t_{b}^{a c t, e}}\left(q_{i}(t)-\overline{q_{i, b}^{a c t}}\right)^{2} \sum_{t=t_{b}^{a c t, s}}^{t_{b}^{a c t, e}}\left(q_{j}(t)-\overline{q_{j, b}^{a c t}}\right)^{2}}},
$$

where $C_{i j, b}^{a c t}$ is the correlation coefficient between the PRs of user $i$ and user $j$ for the $b$ th attraction, $C_{i j, b}^{a c t}$ represents the similarity between the PRVs of user $i$ and user $j . \overline{q_{i, b}^{a c t}}$ represents the mean PR of user $i$ for the $b$ th attraction. The superscript in the equation indicates an attraction experience. $t_{b}^{a c t, s}$ is the start time of the $b$ th attraction. $t_{b}^{a c t, e}$ is the end time of the $b$ th attraction. The correlation coefficient is a normalized covariance value. PR has individual differences. However, since the correlation coefficient is obtained by normalizing the covariance, the influence of individual differences can be reduced. $d_{i j, b}^{a c t}$ is estimated by inputting $C_{i j, b}^{a c t}$ into machine learning $M^{a c t} . d_{i j, b}^{a c t}$ is the degree of friendship after experiencing each attraction. $d_{i j}^{e v}$ is estimated by inputting $\overline{C_{i j, b}^{a c t}}$ into machine learning $M^{e v} . d_{i j}^{e v}$ is the degree of friendship after the offline event. $d_{i j, b}^{a c t}$ and $d_{i j}^{e v}$ represent the degree of friendship as objective variables.

\section{Experiment of Friendship}

The experiment has three purposes as follows. First, it shows that $C_{i j, b}^{a c t}$ is changed by elements other than walking. Second, it shows the relationship among $C_{i j, b}^{a c t}$, favorability, empathy, and friendship. Third, it shows the accuracy of the estimation of friendship when $C_{i j, b}^{a c t}$ and $\overline{C_{i j, b}^{a c t}}$ are input into machine learning.

The experiment was conducted in an amusement park. There are many attractions aimed at making friends in an amusement park. There are also many attractions in which a group of users can experience the attraction at the same time. The users tend to feel positive emotions in an amusement park. We made groups of four people each. The subjects were eight males in their 20 s and four females in their 20 s. 
The subjects in each group were of the same sex. The relationships in the group are shown in Fig. 2. To estimate friendship, there were one friend and two other people met for the first time in each group for each subject. We defined friendships between the friends as CFs and those with the others as SFs. The three groups experienced seven attractions in the same order. In the experiment, we chose an attraction that four people can experience together. Afterwards, the subjects walked to the next attraction. The order of the attractions was set so that the movement distance of the subject was maximum. The order of attractions was a shooting game (act 1), merry-go-round (act 2), video-experience-type attraction (act 3), walk-through-type attraction (act 4), teacup (act 5), riding experience attraction (act 6), and the Ferris wheel (act 7). Act 3 allowed the visitors to watch videos within the attraction. Act 4 allowed the visitors to freely walk around to experience the attraction. Act 6 allowed the visitors to ride on and experience a roller coaster. In all attractions other than act 4 , the subjects were seated while experiencing the attraction.

The subjects were asked to respond to Web questionnaires on favorability and emotions at the end of each attraction. Web questionnaires on favorability and emotions were conducted to verify the effects of empathy and favorability on friendship and $C_{i j, b}^{a c t}$. The questionnaire concerning favorite evocation was "Who was your favorite companion from the end of the previous attraction to the present? (multiple answers allowed)". The questionnaire concerning emotional evocation was "Choose one emotion that was evoked in you by the attraction (pleasant or unpleasant or neither)". A smartphone was used to access the Web questionnaire.

All subjects wore a pulse sensor on their left wrist. We emplayed the wristwatch-type pulsimeter PS-100 made by EPSON as the pulse sensor. The sampling rate was set to 0.25 Hz. Since the Nyquist frequency is $0.125 \mathrm{~Hz}$, waveform in the LF band was obtained. The LF band represents the activity of the sympathetic and parasympathetic nerves. When emotions are evoked, the sympathetic and parasympathetic nerves are activated. Therefore, empathy can be measured with PS-100. The subjects walked to the next attraction. The PR of each subject increases with walking. To analyze the effect of walking on PR during the attraction experience, an acceleration sensor within the smartphone was used. Energy consumption positively correlates with the magnitude of motion and exercise time. ${ }^{(25)}$ The magnitude of each subject's motion can be obtained from the acceleration. A smartphone put in the back pocket of each subject's pants determines the acceleration along three axes. $\alpha_{i}^{\gamma}(t)$ is defined as the acceleration along the $\gamma$-axis of user $i$ at time $t$. $\alpha_{i}^{\gamma}(t)$ changes in accordance with the attitude of the smartphone and the behavior of the user. The attitude of the smartphone depends

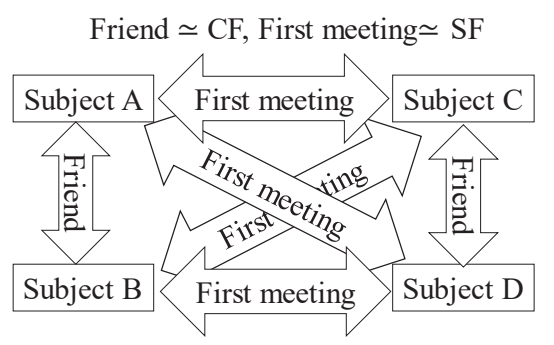

Fig. 2. Group member composition. 
on the shape of the pocket and how the user puts it in the pocket. There is noise in obtaining the movement of the subject. Therefore, we calculate the Euclidean norm $\left|\alpha_{i}(t)\right|$ of $\alpha_{i}^{\gamma}(t)$ as a variable representing motion using

$$
\left|a_{i}(t)\right|=\sqrt{\sum_{\gamma=1}^{3} \alpha_{i}^{\gamma}(t)^{2}},
$$

where $\left|\alpha_{i}(t)\right|$ is independent of the attitude of the smartphone. The acceleration sensor is always loaded with gravitational acceleration. The gravitational acceleration is $9.8 \mathrm{~m} / \mathrm{s}^{2}$. When the subject is not moving, $\left|\alpha_{i}(t)\right|$ is $9.8 \mathrm{~m} / \mathrm{s}^{2}$. Otherwise, $\left|\alpha_{i}(t)\right|$ is greater than $9.8 \mathrm{~m} / \mathrm{s}^{2}$ and the standard deviation is increased. Travel time is determined as $t_{b}^{m v, e}-t_{b}^{m v, s} \cdot t_{b}^{m v, s}$ is the time when the attraction of the $b-1$ th time ended and the movement to the $b$ th attraction started. $t_{b}^{m v, e}$ is the time taken to arrive at the $b$ th attraction. The experience time of the attraction is determined as $t_{b}^{a c t, e}-t_{b}^{a c t, s}$. We used "XPERIA Z3 Compact SO - 02G" made by Sony as the smartphone. The sampling rate was set to $200 \mathrm{~Hz}$. In this experiment, $t_{b}^{m v, s}, t_{b}^{m v, e}, t_{b}^{a c t, s}$, and $t_{b}^{a c t, e}$ were recorded by the experiment supervisor. $t_{b}^{m v, s}$ was the time needed to complete the Web questionnaire. A correlation coefficient of less than 0.2 means no correlation. A correlation coefficient between 0.2 and 0.4 indicates weak correlation. A correlation coefficient between 0.4 and 0.7 means moderate correlation. A correlation coefficient between 0.7 and 1.0 indicates strong correlation.

\section{Relationship between Friendship and Similarity of PRVs}

\subsection{Influence of walking on PRV during attraction experience}

We compare PRs during walking and during the attraction experiences. We clarify the influence of walking on the PR during the attraction experience. We clarify the influence of walking before act 4 on the PR in act 4 .

Figure 3 shows a bar graph of travel time and attraction experience time. Error bars on the bar graph represent the standard deviation. The standard deviation of the PR represents the PRV. The travel time is different for each attraction. The mean experience time and standard deviation of experience time were different for each attraction. Figure 4 shows the PRs during

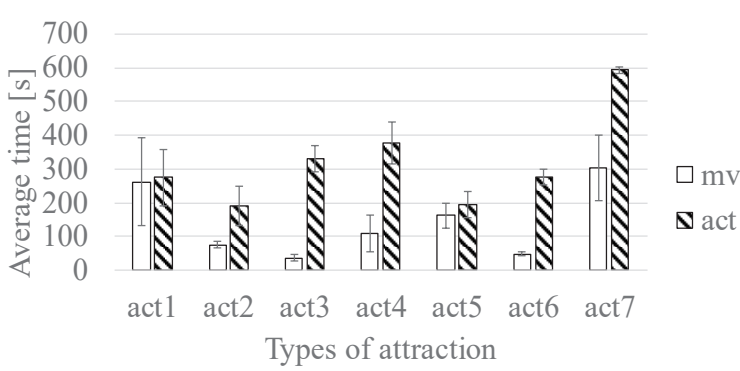

Fig. 3. Travel time and attraction experience time.

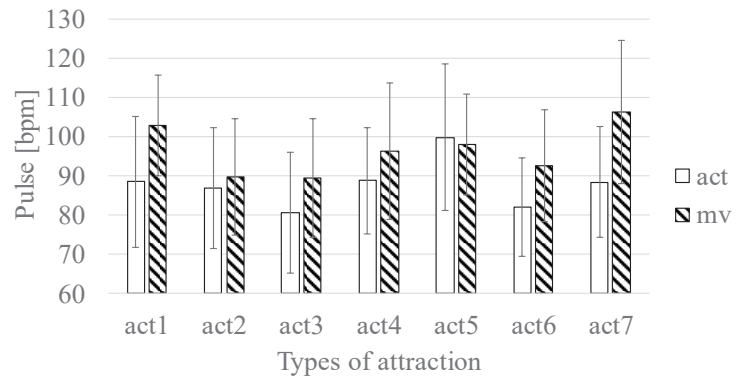

Fig. 4. Pulse during movement and pulse during attraction experience. 
the travel time and during the attraction experience. The mean and standard deviation of PR in act 5 were maximum. Since the users move their hands to rotate the teacup in act 5 , PR increased. The PR in attractions other than act 5 is lower than the PR during walking. The greater the travel time, the greater PR during walking.

Figure 5 shows the mean and standard deviation of the PR of each subject. The standard deviation of PR reflects PRV. There is a difference in the mean PR among subjects. Therefore, it is difficult to evaluate a subject's PR in terms of absolute values. However, compared with the mean PR, the standard deviation of PR shows small individual differences. Covariance is the square of the standard deviation. Therefore, $C_{i j, b}^{a c t}$, which is normalized covariance, is only nominally influenced by individual differences.

To solve this problem, we standardize the PR for each subject and compare the subject's PRs for all attractions in terms of a relative value using

$$
\begin{gathered}
L_{i}^{\beta}(t)=\frac{\left(q_{i}(t)-\overline{q_{i}^{\beta, e v}}\right)}{\sigma_{i}^{\beta}}, \\
\sigma_{i}^{\beta}=\sqrt{\frac{\sum_{t}\left(q_{i}(t)-\overline{q_{i}^{\beta, e v}}\right)^{2}}{N-1}},
\end{gathered}
$$

where $\beta=$ act or mv represents attraction experience or movement, and $\overline{q_{i}^{\beta, e v}}$ is the mean PR during the attraction experience or walking.

The scatter plot of travel time and $L_{i}^{m v}(t)$ is shown in Fig. 6. The correlation coefficient between travel time and $L_{i}^{m v}(t)$ shows moderate correlation. The scatter plot of travel time and the standard deviation of PR during walking is shown in Fig. 7. The correlation coefficient between travel time and the standard deviation of PR during walking shows medium correlation. Figure 8 shows the acceleration during walking and the attraction experience. The acceleration during walking is more than $9.8 \mathrm{~m} / \mathrm{s}^{2}$.

The acceleration during the attraction experience is about $9.8 \mathrm{~m} / \mathrm{s}^{2}$. The travel time affects the PR during the attraction experience. The travel time before the attraction experience and $L_{i}^{a c t}(t)$ are shown in Fig. 9. The correlation coefficient between the travel time before the

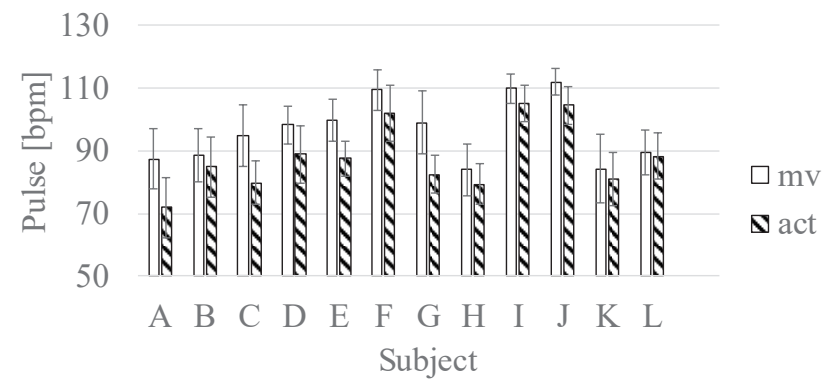

Fig. 5. Average and standard deviation of pulse of each subject.

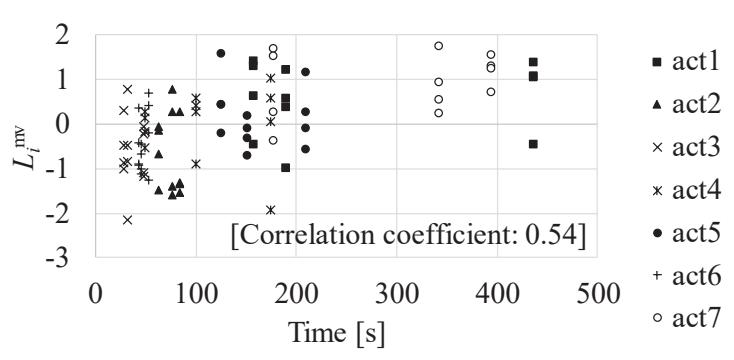

Fig. 6. Travel time and $L_{i}^{m v}(t)$. 


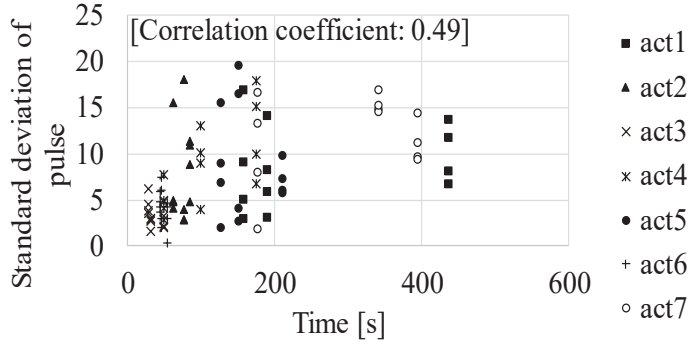

Fig. 7. Travel time and standard deviation of pulse during walking.

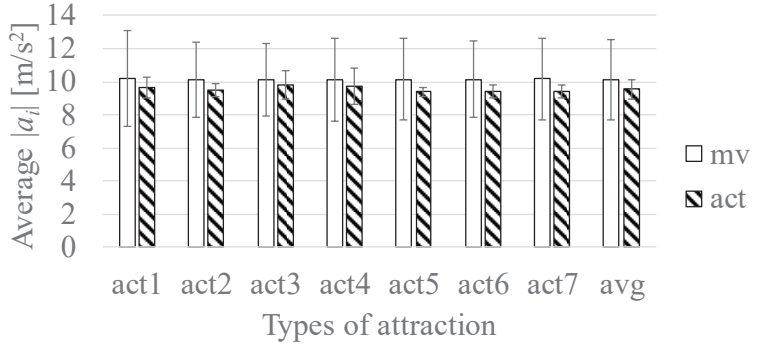

Fig. 8. Acceleration during walking and acceleration during the attraction experience.

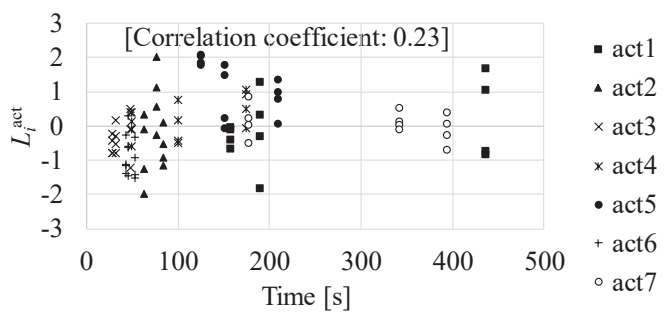

Fig. 9. Travel time before the attraction experience and $L_{i}^{m v}(t)$.

attraction experience and $L_{i}^{a c t}(t)$ shows weak correlation. From the mean and standard deviation in Fig. 7 and the correlation coefficients in Figs. 6 and 9, the increase in the PRV during walking did not affect the PRV during the attraction experience. The travel time before the attraction experience and the standard deviation of the PR during the attraction experience are shown in Fig. 10. The travel time before the attraction experience and the standard deviation of PR during the attraction experience shows no correlation. From the medium correlation between travel time and $L_{i}^{m v}(t)$ and between the standard deviation of travel time and PR, the increase in $L_{i}^{m v}(t)$ has little influence on PRV during the attraction experience. The attraction experience time and $L_{i}^{a c t}(t)$ are shown in Fig. 11. The correlation coefficient between attraction experience time and $L_{i}^{a c t}(t)$ shows no correlation. The attraction experience time and the standard deviation of PR during the attraction experience are shown in Fig. 12. The correlation coefficient between attraction experience time and the standard deviation of PR during the attraction experience shows no correlation. The standard deviation of acceleration during the attraction experience in Fig. 7 was less than one-fifth of the standard deviation of acceleration during walking. From the standard deviation of acceleration in Fig. 7 and the correlation coefficients in Figs. 8, 10, and 11, walking in act 4 did not affect the standard deviation of PR in act 4.

We summarize the above results. Since act 5 requires moving of the hands during the attraction experience, the PR in act 4 was greater than the PR in the other attractions. Walking affects the PR and PRV during walking, and the PR during the attraction experience. However, walking did not affect the PRV during the attraction experience. Walking before act 4 did not affect the standard deviation of the PR in act 4. $C_{i j, b}^{a c t}$ represents the similarity in the PRVs of the users. Therefore, the influence of walking on $C_{i j, b}^{a c t}$ is small. Thus, it is more likely that the similarity in PRVs during the attraction experience is caused by elements other than walking. 


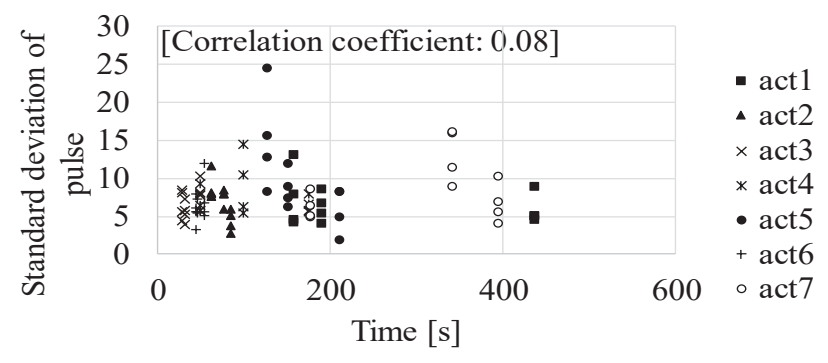

Fig. 10. Travel time before attraction experience and standard deviation of pulse during attraction experience.

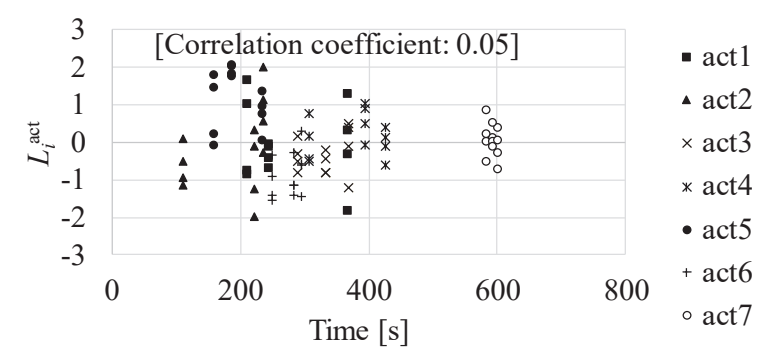

Fig. 11. Attraction experience time and $L_{i}^{a c t}(t)$.

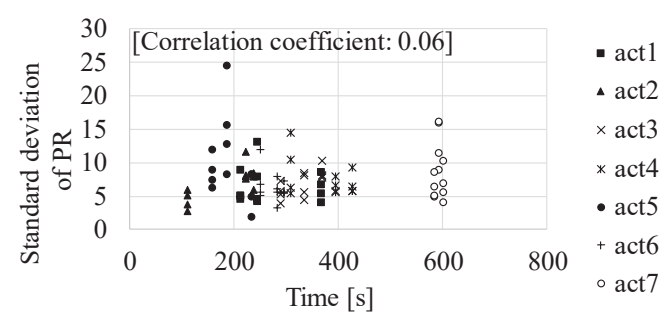

Fig. 12. Attraction experience time and standard deviation of PR during attraction.

\subsection{Relationship between empathy and favorability and $C_{i j, b}^{a c t}$}

We clarify that favorable patterns, favorability rating, and empathy affect $C_{i j, b}^{a c t}$. In this paper, two users liked each other (TL), one of these two users liked a third user (OL), and two users did not like each other (NL); these are defined as the three types of favorable patterns. Table 1 shows the sample size of the favorable pattern in each attraction, taken from Web questionnaire results on favorability.

The mean \pm standard deviation of NL in the sample size per attraction was $2.4 \pm 1.7$, that of OL in the sample size per attraction was $7.1 \pm 2.6$, and that of TL in the sample size per attraction was $8.4 \pm 3.6$. The mean \pm standard deviation of NL was minimum. The mean \pm standard deviation of TL was maximum. The number of occurrences in TL decreased in the order of act $7>$ act $4>$ act $3=$ act $5>$ act $2=$ act $6>$ act 1 . The number of occurrences in OL decreased in the order of act $3=$ act $2>$ act $6>$ act $1>$ act $4=$ act $5>$ act 7 . The number of occurrences in NL decreased in the order of act $5>$ act $6>$ act $1=$ act $2>$ act $4=$ act $3>$ act 7. Table 2 shows the number of emotions evoked during the attraction experience. In act 3 , the number of times negative emotion was evoked was maximum and the number of occurrences in TL was the third highest. In addition, in act 3, the number of occurrences of OL was the second highest. In act 3, the most negative emotion evoked was OL, which was the second most frequently evoked emotion. Therefore, the negative emotion rarely affected the evocation of favorable patterns. We analyzed the influence of empathy on the favorable patterns. When the questionnaires on the evocation of emotion of user $i$ and user $j$ were respectively positivepositive, and negative-negative, empathy (ET) was defined as being evoked. Otherwise, nonempathy (NET) was evoked. 
Table 1

Number of occurrences of favorability for each attraction.

\begin{tabular}{lcrrrccc}
\hline & act1 & act2 & act3 & act4 & act5 & act6 & act7 \\
\hline NL & 3 & 3 & 1 & 1 & 5 & 4 & 0 \\
OL & 7 & 10 & 10 & 6 & 6 & 9 & 2 \\
TL & 8 & 5 & 7 & 11 & 7 & 5 & 16 \\
\hline
\end{tabular}

Table 2

Number of occurrences of emotion for each attraction.

\begin{tabular}{lccccccc}
\hline & act1 & act2 & act3 & act4 & act5 & act6 & act7 \\
\hline Negative & 0 & 0 & 5 & 0 & 2 & 2 & 1 \\
Neutral & 0 & 3 & 5 & 3 & 2 & 4 & 3 \\
Positive & 12 & 9 & 2 & 9 & 8 & 6 & 8 \\
\hline
\end{tabular}

The numbers of occurrences of ET and NET during each altraction experience are shown in Table 3. The mean \pm standard deviation of ET in the sample size per attraction was $9.1 \pm$ 4.6. The mean \pm standard deviation of NET in the sample size per attraction was $8.9 \pm 4.6$. The number of occurrences of ET decreased in the order of act $1>$ act $2=$ act $4>$ act $7>$ act $5>$ act $3>$ act 6 . The number of occurrences of NET decreased in the order of act $6>$ act $3>$ act $5>$ act $7>$ act $2=$ act $4>$ act 1 . The conditional probability of favorability and empathy is calculated as $g(\delta \mid e)$. $e(=\mathrm{ET}, \mathrm{NET})$ represents the type of empathy. $\delta(=\mathrm{NL}, \mathrm{OL}, \mathrm{TL})$ represents the type of favorable pattern. $g(\delta \mid e)$ is shown in Table 4. $g(\mathrm{TL} \mid \mathrm{ET})$ was maximum in $g(\delta \mid \mathrm{ET})$. $g(\mathrm{NET} \mid \mathrm{NL})$ was maximum in $g(\delta \mid \mathrm{NET})$. These results suggest that the number of occurrences of empathy increased when the users liked each other.

We clarify the relationship between the evocation of favorable patterns and $C_{i j, b}^{a c t}$. The mean \pm standard deviation of $C_{i j, b}^{a c t}$ in NL showed no correlation $(0.06 \pm 0.39)$. The mean \pm standard deviation of $C_{i j, b}^{a c t}$ in OL showed a weak correlation $(0.25 \pm 0.41)$. The mean \pm standard deviation of $C_{i j, b}^{a c t}$ in TL showed a weak correlation $(0.29 \pm 0.39)$. We applied multiple comparison tests and show that there are significant differences in the $C_{i j, b}^{a c t}$ of TL, OL, and NC. The sample size of $C_{i j, b}^{a c t}$ in NL was 17. The sample size of $C_{i j, b}^{a c t}$ in OL was 50. The sample size of $C_{i j, b}^{a c t}$ in TL was 59. As a preprocessing for testing the difference between means, the normality test and equality of variances were carried out. D'Agostino's K-squared test (K2 test) was used for the test of normality. The Bartlett test was used to verify the equality of variances. The rejection region was set to 0.1 . From the result of the K2 test, since the $p$-value was 0.12 , the null hypothesis was not rejected. From the result of the Bartlett test, since the $p$-value was 0.97 , the null hypothesis was not rejected. From these results, we used the Tukey-Kramer test as the parametric test to verify the difference between the means of $C_{i j, b}^{a c t}$. Since the $p$-value of $C_{i j, b}^{a c t}$ between NL and OL was 0.19 , the null hypothesis was not rejected. Since the $p$-value of $C_{i j, b}^{a c t}$ between OL and TL was 0.86 , the null hypothesis was not rejected. Since the $p$-value of $C_{i j, b}^{a c t}$ between NL and TL was 0.08 , the null hypothesis was rejected. These results showed that when the users liked each other, the PRVs of the users are similar. Since empathy differs between NL and TL, it is related to the similarity in the PRVs of the users.

We verify the relationship between contact time and $C_{i j, b}^{a c t}$. Contact time is defined as the sum of the attraction experience time and travel time of the users. A scatter plot of contact time 
Table 3

Number of occurrences of empathy for each attraction.

\begin{tabular}{lrrrrrrr}
\hline & act1 & act2 & act3 & act4 & act5 & act6 & act7 \\
\hline ET & 18 & 10 & 5 & 10 & 8 & 4 & 9 \\
NET & 0 & 8 & 13 & 8 & 10 & 14 & 9 \\
\hline
\end{tabular}

Table 4

Conditional probability of favorability and empathy.

\begin{tabular}{lccc}
\hline & \multicolumn{3}{c}{$g(\delta \mid e)$} \\
\cline { 2 - 4 } & NL & OL & TL \\
\hline ET & 0.41 & 0.46 & 0.58 \\
NET & 0.59 & 0.54 & 0.42 \\
\hline
\end{tabular}

and $C_{i j, b}^{a c t}$ is shown in Fig. 13. The correlation coefficient between contact time and $C_{i j, b}^{a c t}$ shows no correlation. The conditional probability of the evocation of favorable patterns during the first half of the amusement park experience and the evocation of favorable patterns during the second half of the amusement park experience are compared. The attractions during the first half are act 1 to act 3 . The attractions during the second half are act 4 to act 7 . The conditional probability of the evocation of favorable patterns for each contact time is calculated using $g(\delta \mid \eta)$. $g(\delta \mid \eta)$ is shown in Table 5. $g$ (OL|first half) is maximum at $g(\delta \mid$ first half). $g$ (TL|first half) is maximum at $g(\delta \mid$ second half $)$. The results indicate that contact time dose not affect $C_{i j, b}^{a c t}$. On the other hand, when contact time is increased, the number of occurrences of TL increases. This suggests that the pattern where one of the two users likes a third user is often evoked when the users feel tension. Note that two of four members in the group met for the first time when the event began. With time, the pattern where two users like each other is often evoked since tension begins to dissipate.

The relationship between the favorability rating and the similarity in the PRVs of users is verified. The favorability rating is calculated using

$$
z_{i j, b}^{a c t}=\sum_{b=1}^{B}\left(u_{i, b}^{a c t}(j)+u_{j, b}^{a c t}(i)\right)
$$

where $z_{i j, b}^{a c t}$ is the favorability rating of user $i$ and user $j$ after the $b$ th attraction, $u_{i, b}^{a c t}(j)$ is a questionnaire result of user $i$ on the favorability of user $j$ after the $b$ th attraction. User $i$ wrote the name of the favorable person on the Web questionnaire. When user $i$ likes user $j, u_{i, b}^{a c t}(j)$ is 1. Otherwise $u_{i, b}^{a c t}(j)$ is 0 . In this work, we classified favorability ratings such that the number of samples would be equal. We defined low favorability rating (LFR) for a relationship between user $i$ and user $j$ where $z_{i j, b}^{a c t}$ is less than or equal to 4 . We defined high favorability rating (HFR) for a relationship between user $i$ and user $j$ where $z_{i j, b}^{a c t}$ is greater than or equal to 5 . The sample size of LFR was 60. The sample size of HFR was 66. A scatter plot of $z_{i j, b}^{a c t}$ and $C_{i j, b}^{a c t}$ is shown in Fig. 14. The correlation coefficient between $z_{i j, b}^{a c t}$ and $C_{i j, b}^{a c t}$ showed weak correlation. The mean \pm standard deviation of $0.17 \pm 0.39$ of $C_{i j, b}^{a c t}$ in LFR showed lack of correlation. The mean \pm standard deviation of $0.31 \pm 0.41$ of $C_{i j, b}^{a c t}$ in HFR showed a weak correlation. We applied the test of the difference between means and showed that there is a significant difference between the 


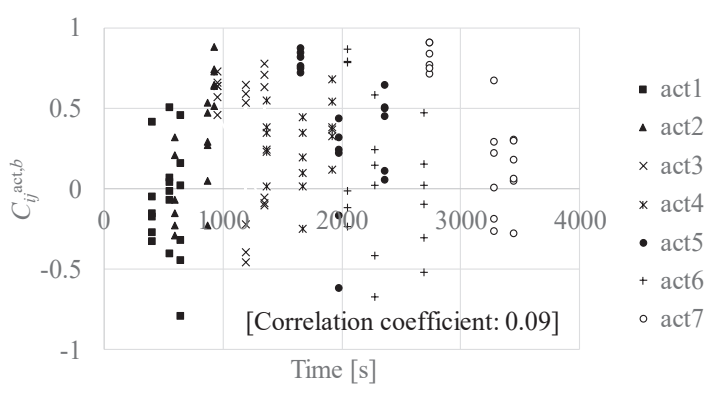

Fig. 13. Relationship between contact time and $C_{i j, b}^{a c t}$.

Table 5

Conditional probability of patterns of favorability for the contact time of each attraction experience.

\begin{tabular}{lccc}
\hline & \multicolumn{3}{c}{$g(\delta \mid \eta)$} \\
\cline { 2 - 4 } & $\mathrm{NL}$ & $\mathrm{OL}$ & $\mathrm{TL}$ \\
\hline First half & 0.13 & 0.5 & 0.37 \\
Second half & 0.14 & 0.32 & 0.54 \\
\hline
\end{tabular}

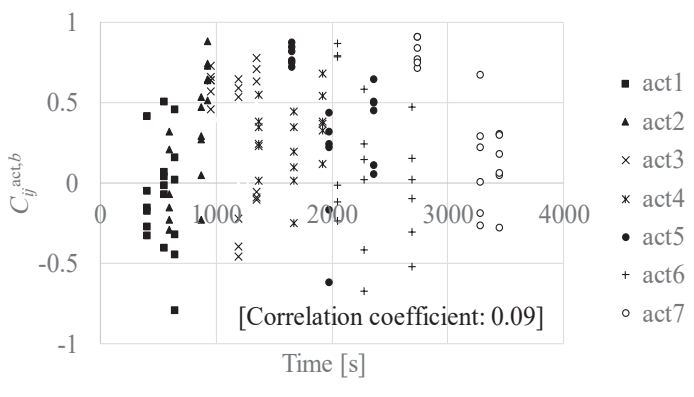

Fig. 14. Relationship between $z_{i j, b}^{a c t}$ and $C_{i j, b}^{a c t}$.

$C_{i j, b}^{a c t}$ of LFR and that of HFR. As a preprocessing for testing the difference between means, a test of normality was carried out. The K2 test was used for the test of normality. The rejection region was set to 0.1 . In the results of this normality test, since the $p$-value was 0.12 , the null hypothesis was not rejected. From this, Welch's $t$-test was used as the parametric test to test the difference between the means of $C_{i j, b}^{a c t}$. In the results of Welch's $t$-test, since the $p$-value was 0.05 , the null hypothesis was rejected. Therefore, there is a significant difference between the mean $C_{i j, b}^{a c t}$ values of LFR and HFR.

We summarize the above results. When the users empathized with each other, the pattern that they liked each other was often evoked. The similarity in the PRVs of the users was large when the users liked each other. Therefore, we suggested that the similarity in the PRVs of the users is increased by empathy. Contact time did not affect the similarity in the PRVs of the users. However, when contact time was increased, the number of occurrences of TL increased. The similarity of PRVs of users at a low favorability rating was uncorrelated with favorability rating. The similarity in the PRVs of the users at a high favorability rating had a weak positive correlation with favorability rating. The similarity in the PRVs of the users differed between LFR and HFR. 


\subsection{Relationship between friendship and $C_{i j, b}^{a c t}$}

We verify the possibility of estimating CFs and SFs from $C_{i j, b}^{a c t}$. The conditional probabilities of friendship and empathy are shown in Table 6. $h(=\mathrm{CFs}, \mathrm{SFs})$ is defined as the type of friendship. The $g(e \mid h)$ values are equal for CFs and SFs. The conditional probabilities of favorability and friendship are shown in Table 7. The $g(\delta \mid h)$ values are equal for CFs and SFs. The results suggest that it is difficult to estimate friendship between user $i$ and user $j$ on the basis of the questionnaire results on favorability and emotions.

For LFR and HFR, we applied the test of the difference in means and showed that there is a significant difference between the $C_{i j, b}^{a c t}$ of CFs and that of SFs. For LFR, the sample size of the $C_{i j, b}^{a c t}$ of CFs was 20, and that of SFs was 40. For HFR, the sample size of the $C_{i j, b}^{a c t}$ of CFs was 22 , and that of SFs was 44 . In the results of testing the normality in the K2 test, since the $p$-value of LFR was 0.17 and the $p$-value of HFR was 0.35 , the null hypotheses were not rejected. Therefore, for HFR and LFR, Welch's $t$-test was used to test the difference in mean $C_{i j, b}^{a c t}$ between CFs and SFs. Table 8 shows the results of Welch's $t$-test. Since the $p$-value of LFR was 0.44 , the null hypothesis was not rejected. Since the $p$-value of HFR was 0.01 , the null hypothesis was rejected.

For TL, ET, and NET with HFR, we applied the test for the difference in means and showed that there was a significant difference in $C_{i j, b}^{a c t}$ between CFs and SFs. For TL with HFR, the sample size of the $C_{i j, b}^{a c t}$ of CFs was 13 and that of SFs was 29. For ET with HFR, the sample size of the $C_{i j, b}^{a c t}$ of CFs was 9 and that of SFs was 19. For NET of HFR, the sample size of the $C_{i j, b}^{a c t}$ of CFs was 13, and that of SFs was 25. In the results of K2 test, since the $p$-value of HFR was 0.35 , the null hypothesis was not rejected. Therefore, for TL, ET, and NET with HFR, Welch's $t$-test was used to verify the difference between the mean $C_{i j, b}^{a c t}$ values. Table 9 shows the test results of the difference between the mean $C_{i j, b}^{a c t}$ values. Since the $p$-value of TL was 0.03 , the null hypothesis was rejected. Since the $p$-value of ET was 0.66 , the null hypothesis was not rejected.

Table 6

Conditional probabilities of empathy and friendship.

\begin{tabular}{lll}
\hline & \multicolumn{2}{c}{$g(e \mid h)$} \\
\cline { 2 - 3 } & \multicolumn{1}{c}{ ET } & NET \\
\hline CFs & 0.5 & 0.5 \\
SFs & 0.51 & 0.49 \\
\hline
\end{tabular}

Table 8

Mean and standard deviation of the favorability rating of $C_{i j, b}^{a c t}$.

\begin{tabular}{lccc}
\hline & \multicolumn{2}{c}{ avg \pm sd } & $p$-value \\
\cline { 2 - 4 } & SFs & CFs & SFs-CFs \\
\hline LFR & $0.20 \pm 0.41$ & $0.12 \pm 0.38$ & 0.44 \\
HFR & $0.23 \pm 0.44$ & $0.47 \pm 0.31$ & 0.01 \\
\hline
\end{tabular}

Table 7

Conditional probabilities of favorability and friendship.

\begin{tabular}{lccc}
\hline & \multicolumn{3}{c}{$g(\delta \mid h)$} \\
\cline { 2 - 4 } & NL & OL & TL \\
\hline CFs & 0.13 & 0.39 & 0.48 \\
\hline SFs & 0.14 & 0.4 & 0.45 \\
\hline
\end{tabular}

Table 9

Difference between the mean values for $C_{i j, b}^{a c t}$ for TL, ET, and NET of CFs and SFs with HFR.

\begin{tabular}{lccc}
\hline & \multicolumn{2}{c}{ avg \pm sd } & $p$-value \\
\cline { 2 - 4 } & SFs & CFs & SFs-CFs \\
\hline TL & $0.26 \pm 0.41$ & $0.52 \pm 0.31$ & 0.03 \\
ET & $0.43 \pm 0.39$ & $0.5 \pm 0.42$ & 0.66 \\
NET & $0.08 \pm 0.42$ & $0.45 \pm 0.22$ & 0.001 \\
\hline
\end{tabular}


Since the $p$-value of NET was 0.001 , the null hypothesis was rejected. To explain why there is no significant difference in ET, we measured the evocation of emotions during the attraction experiences. In Table 6, there is no difference between CFs and SFs in the number of times empathy is evoked during the attraction experience. In CFs, the mean $C_{i j, b}^{a c t}$ of NET and that of ET were equal.

We summarize the above results. The favorability ratings of CFs and SFs and the number of times empathy is evoked in CFs and SFs during the attraction experience were equal. From the data in Tables 6 and 7, we conclude that it is difficult to estimate friendship between user $i$ and user $j$ from the questionnaire results on favorability and emotions. When the favorability rating of the users is high, the PRVs of the users are similar since the users can ease them. Therefore, $C_{i j, b}^{a c t}$ can be used to estimate friendship evoked during each attraction experience. Since there was a difference in the $C_{i j, b}^{a c t}$ with HFR, $\overline{C_{i j, b}^{a c t}}$ can be used to estimate friendship evoked at an offline event.

\section{Evaluation of Estimation of Friendship Evoked at Each Attraction and Offline Event}

We input $C_{i j, b}^{a c t}$ into the linear regression model and estimate $z_{i j, b}^{a c t}$. We show the estimation accuracy for friendship evoked at each attraction when $C_{i j, b}^{a c t}$ was input into $M^{a c t}$ during the $b$ th attraction experience. We show the estimation accuracy for friendship evoked at an offline event for $\overline{C_{i j, b}^{a c t}}$ input into $M^{e v}$.

In Sect. 5, we showed that it is possible to estimate CFs and SFs from the $C_{i j, b}^{a c t}$ with HFR. Therefore, as a preliminary estimation of $\mathrm{CFs}$ and $\mathrm{SFs}$, we estimated the favorability rating of the users. The linear regression model was constructed by inputting $z_{i j, b}^{a c t}$ as an objective variable and $C_{i j, b}^{a c t}$ as an explanatory variable using

$$
\hat{z}_{i j, b}^{a c t}=a C_{i j, b}^{a c t}
$$

where $\hat{z}_{i j, b}^{a c t}$ is the estimated $z_{i j, b}^{a c t}$ and $a$ is the coefficient of the explanatory variable. The sample size was 126 . In the linear regression model, $R^{2}$ was 0.29 and $a$ was 6.78 . We set up the null hypothesis of "a is 0 " for the coefficients of the linear regression model and carried out Welch's $t$-test. Since the $p$-value was $9.32 \times 10^{-11}$, the null hypothesis was rejected. Therefore, $C_{i j, b}^{a c t}$ is the effective explanatory variable for estimating $z_{i j, b}^{a c t}$.

We performed a 3-fold cross-validation with one group as a test set and the other groups as the training set and evaluated the estimation accuracy of friendship evoked at each attraction. The sample size of CFs for each attraction was 6 and the sample size of SFs for each attraction was 12. Therefore, the sample size of the test set was 42 and the sample size of the training set was 84 . We estimated friendship evoked at each attraction using machine learning applications such as Random Forest, Naive Bayes, linear kernel SVM, and Gauss kernel SVM. We used R to implement machine learning. Random Forest was implemented using the Random Forest package of R. Naive Bayes, linear kernel SVM, and Gaussian kernel SVM were implemented using an e1071 package of $\mathrm{R}$. The parameters of machine learning were set to the default values 
of each package. Recall, precision, and $f$-measure were used in the evaluation. The $f$-measure $F$ was calculated as

$$
F=\frac{2 R P}{R+P}
$$

where $R$ is the recall, $P$ is the precision, and $F$ is the $f$-measure.

Table 10 shows the results of using Random Forest since it yielded the maximum estimation accuracy for friendship. It was shown that the evocation of friendship at each attraction can be estimated with the $f$-measure, which had a maximum of 0.74 . In the estimation results of acts 1 and 2, when the favorability rating of the users was low, the $f$-measure was small. When the favorability rating of the users was high, the $f$-measure for the all attractions other than act 5 was large. The degree of this correlation was higher than a random occurrence. From the estimation results, it is shown that accurate estimation can be achieved using the similarity in the PRVs of the users with HFR. The reason why the estimation accuracy for act 5 was small was that $C_{i j, b}^{a c t}$ in act 5 included PRV, which was increased by exercise.

We performed a 3-fold cross-validation with one group used as the test set and the other groups as the training set and evaluated the estimation accuracy for friendship at the offline event. The sample size of CFs for each attraction was 6, and the sample size of SFs for each attraction was 12 . Therefore, the sample size of the test set was 6 , and the sample size of the training set was 12 . We estimated the friendship evoked at each attraction using a machine learning application such as Random Forest, Naive Bayes, linear kernel SVM, and Gauss kernel $\mathrm{SVM}$. We used $\mathrm{R}$ to implement machine learning. The parameters of machine learning were set to the default value of each package. Recall, precision, and $f$-measure were used in the evaluation.

Table 11 shows the results of using Random Forest since it yielded the maximum estimation accuracy for friendship. It was shown that the evocation of friendship at offline events has an estimated mean $f$-measure of 0.78 .

Table 10

Estimation result of friendship evoked at each attraction using $M^{a c t}$.

\begin{tabular}{llllllll}
\hline & act1 & act2 & act3 & act4 & act5 & act6 & act7 \\
\hline $\mathrm{R}$ & 0.29 & 0.5 & 0.71 & 0.63 & 0.38 & 0.58 & 0.75 \\
$\mathrm{P}$ & 0.31 & 0.5 & 0.69 & 0.63 & 0.38 & 0.58 & 0.72 \\
$\mathrm{~F}$ & 0.3 & 0.5 & 0.7 & 0.63 & 0.38 & 0.58 & 0.74 \\
\hline
\end{tabular}

Table 11

Estimation result of friendship evoked at offline event using $M^{e v}$.

\begin{tabular}{lccccc}
\hline & CFs & SFs & R & P & F \\
\hline CFs & 5 & 3 & 0.83 & 0.63 & 0.71 \\
SFs & 1 & 9 & 0.75 & 0.9 & 0.82 \\
\hline
\end{tabular}




\section{Contribution and Limitation}

The experimental results showed that friendship can be estimated from the similarity in the PRVs of the users during the attraction experience. In a related work, friendship was measured using a questionnaire and large equipment such as an electroencephalograph and electrocardiogram, which placed economical and physical burdens on the user. There is, as yet, no research on easily estimating friendship evoked at an offline event in the real world. Such research is challenging and innovative. The proposed method is inexpensive and compact and can be easily popularized through the use of a wristwatch-type pulsimeter. The proposed method can identify the place, time, and target of the evoked friendship. Therefore, the proposed method is effective and useful. We showed that there is a weak positive correlation between the favorability rating and the similarity in PRVs during the attraction experience. We showed that the similarity in the PRVs of CFs is higher than that of SFs when the favorability rating is high and the users feel favorably toward each other. We suggested that similarity in the PRVs of the users is increased by empathy. This work contributes to clarifying the effects of friendship, favorability, and empathy on the similarity in PRVs.

However, to use the proposed method, two conditions must be satisfied. First, since the accuracy of estimation was significantly decreased by the momentum of the attraction, it is necessary to measure PR in an environment with low momentum. Therefore, outdoor events with high momentum, such as sports and dance parties, are unsuitable. When indoor events with low momentum such as games and movie theaters, we can estimate friendship by the proposed method. Second, since the estimation accuracy for friendship with LFR was low, it is necessary to prepare an ice breaker at the event to ease tension. When these two conditions are satisfied, the proposed method can be used to estimate friendship with high accuracy.

\section{Conclusions and Future Works}

We proposed a method of estimating friendship using the similarity in the PRVs of users. The influence of walking on $C_{i j, b}^{a c t}$ was small. When the users empathize with each other, they like each other. The influence of contact time on the similarity in the PRVs of users was small. However, when the contact time was increased, the number of occurrences of TL also increased. The correlation coefficient between the similarity of the PRVs of the users and the favorability rating shows a weak positive correlation. When favorability rating of the users is high, the PRVs of the users are similar since the users are relaxed. We can estimate $z_{i j, b}^{a c t}$ using the linear regression model with a weak explanatory power $R^{2}$. We showed that friendship evoked during the attraction experience can be estimated to have an $f$-measure of 0.74 at maximum from the similarity in the PRVs of the users for each attraction and that the evocation of friendship at an offline event can be estimated from the mean of the similarity in the PRVs of the users for all the attractions with a mean $f$-measure of 0.78 . It is difficult to estimate friendship between user $i$ and user $j$ from the questionnaire results on favorability and emotions.

In this research, we conducted experiments in which men and women in their 20s experience attractions in an amusement park. We verified the usefulness and effectiveness of our proposed 
method. We will investigate the factors behind the differences in the similarity of PRVs between friends and newly met people. In addition, we will measure the accuracy of friendship estimation taking age into consideration. We will apply the proposed method to other events and confirm its usefulness.

\section{References}

1 C. M. Ridings and D. Gefen: J. Comput.-Mediated Commun. 10 (2004) 10110.

2 V. A. Zeithaml: American Marketing Association First Services Marketing Conf. (1981) 186-190.

3 R. Du, Z. Yu, T. Mei, Z. Wang, Z. Wang, and B. Guo: Proc. 2014 ACM Int. Joint Conf. Pervasive and Ubiquitous Computing (2014) 425-434.

4 A. Q. Macedo, L. B Marinho, and R. L. Santos: Proc. 9th ACM Conf. Recommender Systems (2015) 123-130.

5 M. Wu, Z. Wang, H. Sun, and H. Hu: 2016 3rd Int. Conf. In Information Science and Control Engineering (ICISCE). (2016) 379-385.

6 Z. Wang, J. Liao, Q. Cao, and H. Qi, Z. Wang: IEEE Trans. Mobile Comput. 14 (2015) 538.

7 R. G. Adams, R. Blieszner, and B. DeVries: J. Aging Studies 14 (2000) 117.

8 P. M. Sias and D. J. Cahill: Western J. Commun. (includes Communication Reports) 62 (1998) 273.

9 X. Liu, Q. He, Y. Tian, W. C. Lee, J. McPherson, and J. Han: Proc. 18th ACM SIGKDD Int. Conf. Knowledge Discovery and Data Mining (2012) 1032-1040.

10 D. Buhrmester: Child Dev. 61 (1990) 1101.

11 M. J. Mendelson and F. E. Aboud: Canadian J. Behavioural Sci./Revue Canadienne Des Sciences du Comportement 31 (1999) 130.

12 M. H. Davis: J. Personality Soc. Psychology 44 (1983) 113.

13 Y. Motomura, A. Takeshita, Y. Egashira, T. Nishimura, Y. K. Kim, and S. Watanuki: J. Physiol. Anthropol. 34 (2015) 13.

14 P. L. Jackson, A. N. Meltzoff, and J. Decety: Neuroimage 24 (2005) 771.

15 C. Y. Yang, J. Decety, S. Lee, C. Chen, and Y. Cheng: Brain Res. 1251 (2009) 176.

16 J. Decety and K. J. Michalska: Dev. Sci. 13 (2010) 886.

17 H. Fukushima, Y. Terasawa, and S. Umeda: Int. J. Psychophysiology 79 (2011) 259.

18 S. D. Kreibig: Biol. Psychol. 84 (2010) 394.

19 M. J. Hove and J. L. Risen: Soc. Cognit. 27 (2009) 949.

20 S. H. McFadden, A. M. Knepple, and J. A. Armstrong: J. Religious Gerontology 15 (2003) 39.

21 R. Rakshit, V. R. Reddy, and P. Deshpande: Proc. 2nd Workshop on Emotion Representations and Modelling for Companion Systems (2016) 2.

22 L. K. Cirelli, S. J. Wan, and L. J. Trainor: Infancy 21 (2016) 807.

23 R. Zhao, T. Sinha, A. W. Black, and J. Cassell: Int. Conf. Intelligent Virtual Agents (2016) 218-233.

24 M. Brambilla, S. Sacchi, M. Menegatti, and S. Moscatelli: J. Nonverbal Behav. 40 (2016) 171.

25 M. Y. Zarrugh, F. N. Todd, and H. J. Ralston: Eur. J. Appl. Physiol. Occupational Physiol. 33 (1974) 293.

26 N. Eagle, A. S. Pentland, and D. Lazer: Proc. National Academy of Sciences 106 (2009) 15274.

\section{About the Authors}

Yusuke Kajiwara received his M.E. degree from Kanazawa University in 2011, and his Ph.D. degree from Graduate School of Natural Science and Technology, Kanazawa University, Japan, in 2013. He was an assistant professor of Information Science and Technology, Ritsumeikan University, Japan, in 2013. Currently, he is an associate professor in the Department of Production Systems Engineering and Sciences, Komatsu University.

Yuki Kubo is a student of Graduate School of Information Science and Engineering, Ritsumeikan University. 
Haruhiko Kimura completed the doctoral program at Graduate School of Engineering, Tohoku University, in 1979 (Doctor of Engineering). He joined Fujitsu Ltd. in the same year. He became a lecturer at Kanawaza Woman's College in 1980, an associate professor at School of Economics, Kanazawa University, in 1984, and then a professor at Graduate School of Natural Science and Technology, Kanazawa University. Currently, he is a professor in the Department of Production Systems Engineering and Sciences, Komatsu University. His research interest is in applications of soft computing. He is a member of the Japanese Society for Artificial Intelligence, and the Information Processing Society of Japan. 